\title{
Complex Nodule Shape
}

National Cancer Institute

\section{Source}

National Cancer Institute. Complex Nodule Shape. NCI Thesaurus. Code C157677.

A complex shape has no normally geometric shape and combines multiple more simple shapes. If no classically defined shape applies, the lesion is likely complex. 